\title{
Analysis of DRL responding by rats with septal damage
}

\author{
PAUL ELLEN and WILLIAM C. AITKEN, JR.*† \\ Georgia State University, Atlanta, Georgia 30303
}

\begin{abstract}
Normal rats and rats with septal lesions were exposed to several DRL schedules having different delay requirements. Response output was an inverse function of delay requirement, with septals responding more than normals on each schedule. Responding by septals depended upon the reinforcement generated by each schedule, while that of normals was additionally affected by the performance established by the previously run schedule. Since septals could acquire a temporal discrimination on DRL 10 but not on longer DRL schedules, it was concluded that the absence of temporal discrimination by septals on the longer DRL schedules is secondary to a failure to suppress short latency responses.
\end{abstract}

In a previous study (Ellen \& Aitken, 1971), we demonstrated that animals with septal lesions failed to acquire a temporal discrimination on a DRL 20 schedule. For these animals, the response probabilities (IRTs/OP) of nonreinforced responses remained constant at all values of delay since the previous response. In contrast, normal animals developed an increase in response probability as the required delay was approached. The present experiment is concerned with assessing the generality of this observation. That is, do septal animals fail to show temporal discrimination at shorter values of DRL requirement, and what are the upper limits of such discrimination in the case of normal animals?

\section{METHOD}

\section{Subjects and Apparatus}

Eight male Long-Evans hooded rats, housed individually before and throughout the study, served as Ss in this experiment. Ss averaged $400 \mathrm{~g}(390-426 \mathrm{~g})$ in weight at the start of the experiment and $316 \mathrm{~g}(272-361 \mathrm{~g})$ at surgery, the latter weight representing approximately $78 \%$ of ad lib (preexperimental) weight. Four rats received septal lesions, while four animals served as unoperated controls. Ss were run in two squads of four rats each in four Scientific Prototype operant chambers linked to an IBM-1800 data acquisition and control system, which programmed the session and reinforcement schedules. This system recorded responses as interresponse times (IRTs) for each animal and was also used to subsequently gate the IRTs into 1-sec bins (see Ellen \& Aitken, 1971, for a more detailed account of this system).

\section{Behavioral Procedures}

All Ss were trained to barpress for standard $45-\mathrm{mg}$ food pellets (Noyes) and allowed to obtain a minimum of 200 (usually 200-300) reinforcements immediately upon acquisition of the barpressing habit. Ss received 10 days of continuous

\footnotetext{
*A version of this paper was read at the XII Annual Meeting of the Psychonomic Society, November 1971, St. Louis, Missouri.

†The authors wish to acknowledge the assistance of Mr. John Braggio in the performance of histological and some of the statistical analyses.
}

reinforcement (CRF) pretraining, followed by exposure to the six DRL (differential reinforcement of low rates) schedules. CRF was started on the day following completion of bar training and continued each day until each $S$ received 150 reinforcements (usually 10-20 min). Surgery was performed on the day between the fifth and sixth day of CRF for the septal group, while normals received 10 consecutive days of CRF pretraining. DRL runs lasted $50 \mathrm{~min}$ on each experimental day. Throughout pretraining and the remainder of the experiment, all Ss received a supplementary ration (i.e., in addition to food pellets received as reinforcement) of $15-20 \mathrm{~g}$ of ground laboratory rat chow made up into a wet mash for approximately $50 \mathrm{~min}$ following each session. Thus all Ss were maintained on a 22-h food deprivation schedule throughout the experiment (on run as well as on nonrun days).

Each of the six DRL schedules was run for 15 days in the following order: DRL $30,40,30,10,50,20$. Each rat in a run squad ran on the same schedule. The 1800 system at the time this study was implemented lacked the flexibility (Ellen, DeLoache, \& Bonds, 1972) to run a counterbalanced design of DRL schedule values within the same run. Accordingly, the influence of order effects in the running of the various schedules produces a confounding in the results, a factor to be taken into account in interpreting the findings of this study.

All Ss were run 5 days a week for 3 weeks on each schedule. The transition day for changing the DRL value was the day following Day 15 of each schedule. On the transition day, Ss were run for half the session $(25 \mathrm{~min})$ on the same schedule in effect for the previous 15 sessions, and the latter half of the session $(25 \mathrm{~min})$ under the new schedule contingency. The change in DRL value was unsignaled to the animals. Ss were run on the new schedule for 15 sessions, with the first full day of DRL following the transition day being counted as Day 1.

\section{Surgery and Histology}

Animals receiving septal lesions received sodium pentobarbital (Nembutal) anesthesia (40 mg/kg body weight, IP), along with $0.5 \mathrm{cc}(0.1 \mathrm{mg})$ atropine sulfate to reduce respiratory distress. Local infiltration of xylocaine hydrochloride $(2 \%)$ with epinepherine was used during surgery to supplement the Nembutal anesthesia and to reduce bleeding. Each experimental animal received $0.2 \mathrm{cc}$ procaine penicillin $G(60,000$ units, Duracillin) postoperatively.

A midline incision was made, the skull exposed, and a hole drilled at the appropriate coordinate with a dental drill. Anodal electrolytic lesions $(2 \mathrm{~mA}, 20 \mathrm{sec})$ were made by a Stoelting 58040 electrolytic lesion maker, utilizing a Baltimore Instrument Company stereotaxic instrument for stereotaxic orientation. Lesion coordinates were taken from the rat brain atlas of Pellegrino and Cushman (1967). Midline septal lesions were 


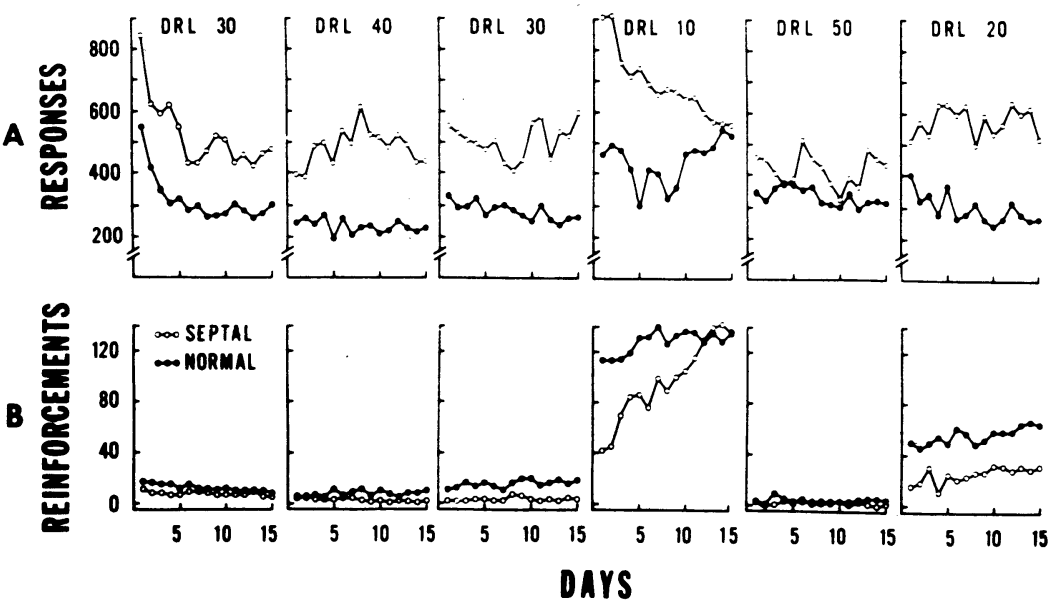

Fig. 1. Responses emitted (A) and reinforcements received (B) by normal rats and rats with septal lesions on DRL 10, 20, 30,40 , and 50 , in order of run. placed $7.8 \mathrm{~mm}$ anterior to and $6.5 \mathrm{~mm}$ above the interaural line.

Following completion of the experiment, the septal animals were sacrificed with a lethal dose of Nembutal, perfused intracardially with Ringer's saline and formalin solutions, and the brains were fixed in the formalin. Serial paraffin sections 40 microns thick were used to aid in lesion localization. The lesions were generally posterior, with the maximum extent being at the level of the anterior commissure, and no evidence of damage to anterior septum or diagonal band was found. There was evidence of damage extending to the plane of the hippocampal commissure and the descending columns of the fornix. None of the lesions involved thalamic structures. Dorsally there was slight damage to overlying corpus callosum in a few instances. "Rage" behavior was evident in all of the animals to varying degrees for a period of several days postoperatively. However, examination of the performance of individual animals revealed no correlation of the "rage" behavior and any of the measures of DRL performance.

\section{RESULTS}

\section{Responses and Reinforcements}

Figure 1 ( $\mathrm{A}$ and $\mathrm{B}$ ) presents responses emitted and reinforcements received on each of the DRL schedules run during the course of the experiment. A three-factor analysis of variance (Winer, 1962), with repeated measures on the within-Ss factors of schedules and training (days), revealed that for both responses (R) and reinforcements (Rf) there were significant schedules effects $(R: F=5.11, p<.01 ; R f: F=41.62, p<.01)$, days effects $(R: F=5.75, p<.01 ; R f: F=6.66$, $\mathrm{p}<.01)$, and Schedules by Days interactions effects ( $R$ : $\mathrm{F}=1.80, \mathrm{p}<.01 ; \mathrm{Rf}: \mathrm{F}=3.12, \mathrm{p}<.01)$. While main effects for lesions were not found to be significant on either of the response or reinforcement measures, the three-factor interaction (i.e., Lesions by Schedules by Days) was significant for both measures ( $R: F=1.44$, $\mathrm{p}<.05$; Rf: $\mathrm{F}=1.64, \mathrm{p}<.01)$. Thus, it would appear that the effects of schedules and days on both responding and reinforcement depended on the lesion and that the relatively strong effects of schedules and days and their interaction masked lesion main effects.

Accordingly, a Newman-Keuls test (Winer, 1962) of all possible paired comparisons of lesions and schedules was performed, the results of which are summarized in Fig. 2 (A and B) for responses and reinforcements, respectively. Septally lesioned rats responded more than normal control animals on each schedule $(\mathrm{p}<.01)$.

Further, even though the schedules were not run in a counterbalanced order among individual animals, it is nevertheless clear that for both septals and normals there is a decline in responding as the DRL requirement is increased. Responding of septal animals on DRL 10 was significantly higher, while responding on DRL 50 was significantly lower than that on DRL 20, 30 (1 and 2), or $40(\mathrm{p}<.01)$ schedules. Additionally, responding on DRL 20 was significantly more than on DRL 30 (2) and DRL 40. In the case of the normal animals, response rates on DRL 20,30, or 50 did not differ from each other, while responding on DRL 10 was significantly higher and that on DRL 40 was significantly lower $(\mathrm{p}<.01)$ than the rates generated by DRL 20,30, or 50 .

When the reinforcement data (Fig. 2-B) are examined, it can be seen that septal animals receive significantly less reinforcement than normals on DRL 10 and 20 $(p<.01)$. On the other schedules, no differences between the septals and normals appeared. However, both normal and septal animals obtained more reinforcement on DRL $10(\mathrm{p}<.01)$ than on any of the other schedules. Normals also received significantly more reinforcements on DRL $20(p<.01)$ than on any of the remaining schedules, while, in the case of the septal animals, the remaining schedules (i.e., DRL 20, 30, 40, and 50) produced essentially the same number of reinforcements.

\section{Conditional Response Probabilities}

Interresponse times (IRTs) and conditional response probabilities (IRTs/OP, Anger, 1956) were calculated for the last 3 days that each schedule (with the exception of the first DRL 30 schedule) was in effect. These were gated into $1-\mathrm{sec}$ bins, thereby allowing direct comparisons of the IRT or the response probability for any value of delay up to $60 \mathrm{sec}$.

For both normal and septal animals, the IRTs on each 
Fig. 2. Responses (A) and reinforcements (B) as a function of DRL requirement (averaged over the 15 days each schedule was in effect).
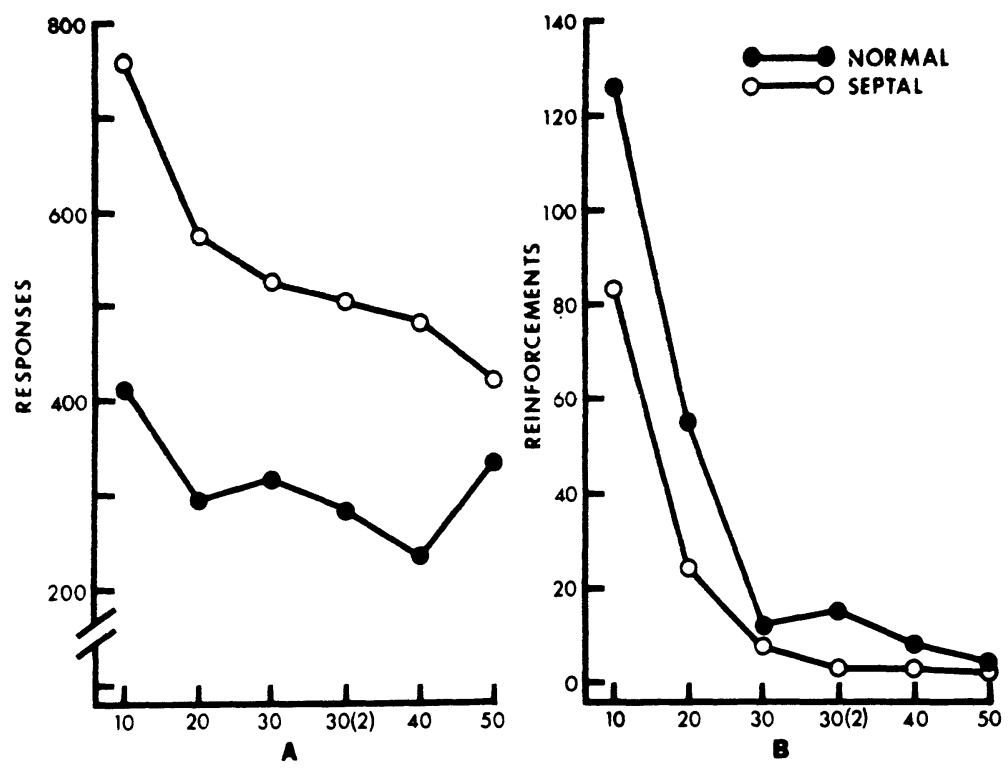

DRL SCHEDULE

schedule, with the exception of DRL 10, were unimodal, with the mode occurring at a delay of $1-2 \mathrm{sec}$. On DRL 10 , both normal and septal rats showed a bimodal distribution, with a second mode occurring in the 10- to 11 -sec range.

The conditional response probability (IRTs/OP) at any delay from a previous response is defined as the probability of a response given that the $S$ has waited at least this long between responses, and is intended to take into account the unequal opportunity of different delays within a session following the occurrence of other delays. Random responding with respect to time yields a constant IRT/OP function (i.e., a straight horizontal line) for all values of delay, and any deviation from this constant function is taken to indicate some form of temporal discrimination. The IRTs were converted to IRTs/OP scores for delays up to $60 \mathrm{sec}$ from a previous response. Figure 3 presents IRTs/OP scores of septal and normal animals for the first $30 \mathrm{sec}$ of delay. On DRL 10, the IRTs/OP curves for both septal and normal animals are quite similar for delays of less than $10 \mathrm{sec}$ (before vertical line in Fig. 3). However, in the range of reinforced delays (i.e., delays of up to $8 \mathrm{sec}$ beyond reinforcement), septal animals show higher response probabilities on this schedule than do normals (Kruskal-Wallis, $\mathrm{H}=11.14, \mathrm{p}<.01$ ). For DRL requirements of 20,30 , or $40 \mathrm{sec}$, the response probabilities for less-than-required delays were higher for septals than for normals (DRL 20, $\mathrm{H}=7.3, \mathrm{p}<.01$; DRL 30, $\mathrm{H}=6.7, \mathrm{p}<.01 ;$ DRL $40, \mathrm{H}=7.2, \mathrm{p}<.01$ ).
Fig. 3. Comparison of conditional response probabilities (IRTs/OP) of normal and septal rats on DRL 10 (closed and open triangles, respectively) and the medians of DRL 20, 30, 40, and 50 (closed and open circles).

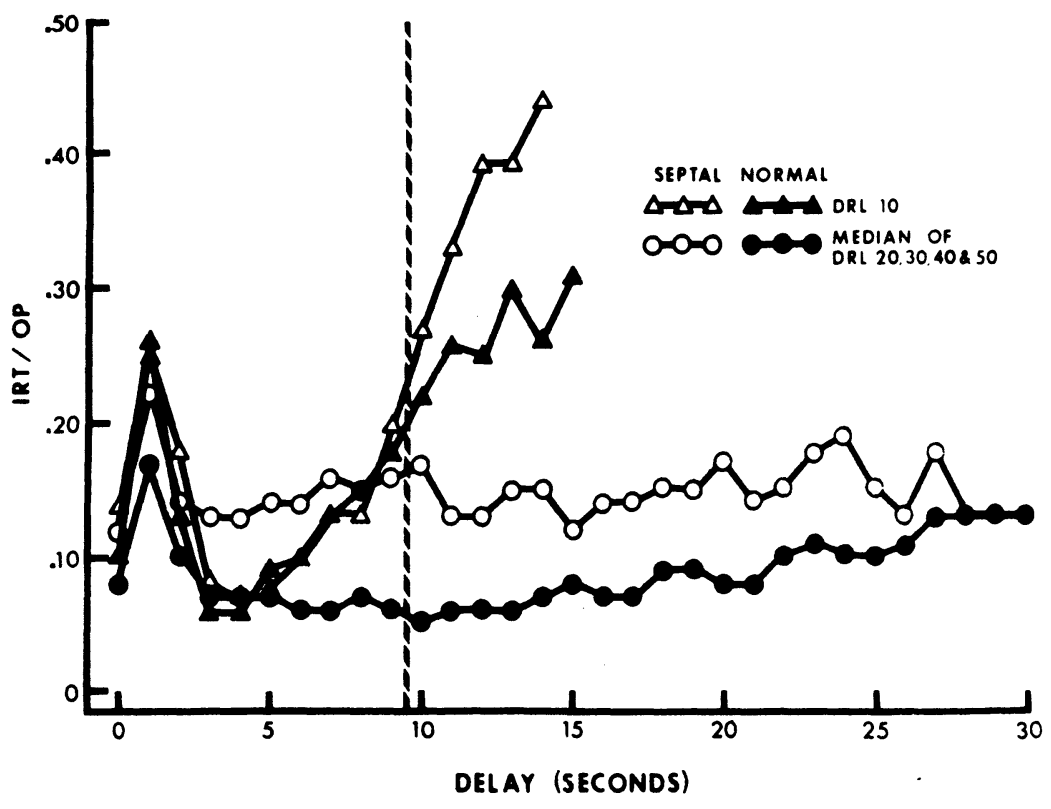


On DRL 50, there was no difference between the response probabilities of septals and normals. However, the most important observation was the fact that, despite the difference between septal and normal animals on DRL 20,30, and 40, there was no difference between DRL 20,30,40, and 50 within each of the two lesion groups. A two-factor analysis of variance with repeated measures on within-group factors of schedules and delays between responses (bins) disclosed no significant differences (septals: $F=1.69, \quad p>.05$; normals: $F=2.22, p>.05$ ). Accordingly, the medians of the probabilities generated by the DRL 20,30,40, and 50 schedules at each delay from the previous response were computed for each group and are also plotted in Fig. 3. From this figure, it will be noted that the median probability at each delay for the septal animals is different from the normals. Following an initial peak at a delay of $2 \mathrm{sec}$, septal animals show a decrease in probability to a level in the .14-.16 range, and this probability level remains constant at all delays beyond this point. In contrast, the curve for the normal animals displays a different trend. After the initial peak, the response probability decreases to a level in the $.05-.06$ range but does not remain constant. After a delay of approximately $14 \mathrm{sec}$, there is a gradual increase in response probability to the level of the septal animals. This occurs between 27-30 sec after a previous response. Beyond $30 \mathrm{sec}$ of delay, response probabilities for both groups become variable and random.

It is of further interest to compare the two DRL 10 curves with the median curves of both groups. It will be noted that in the case of the normal animals, following the low point in response probability (immediately after the initial peak) the median probability curve lies below the curve generated by DRL 10 over the entire range of delays. In the case of septal animals, the median probability curve is above the DRL 10 curve for short delays $(2-9 \mathrm{sec})$.

\section{DISCUSSION}

It is clear from the results of this study that, while septals differ from normals in the amount of responding generated by a DRL schedule, nonetheless for both groups the function relating response output to schedule requirement is essentially the same. That is, with low DRL requirements generally more responses are generated than with higher DRL requirements. This finding is similar in many respects to one reported by Staddon (1965). Staddon found that response rate of pigeons on DRL values of $5,10,15,20$, and $30 \mathrm{sec}$ was an approximately linear inverse function of DRL requirement, with the linearity breaking down somewhere between DRL 22 and DRL 30.

Of particular interest is the fact that the simple inverse relationship found in the present study does not seem to hold as strictly in the case of normal animals as it does for the septals. It will be recalled that, in the case of the normal animals, the rate generated by DRL 50 was in fact higher than that generated by DRL 40 . This departure from a simple inverse relationship most likely reflects confounding due to the order in which the schedules were run. It will be recalled that DRL 50 was run after DRL 10. This effect is also found in the data of the DRL 30 schedules. The rate generated by the first exposure to DRL 30 was higher, although not statistically significant, than the rate generated by the second DRL 30. In this instance, the first DRL 30 followed CRF pretraining. Thus, in both instances where there was a change from a schedule producing a high reinforcement density to one producing a relatively low density of reinforcement (i.e., from DRL 10 to DRL 50 and from CRF to DRL 30), the rate on the latter schedule is apparently elevated above what it would have been were the immediately previous reinforcement history not a factor. This would imply that in the performance of normal animals on a given DRL schedule there is a residual influence of the previous reinforcement history, as well as the influence of the current schedule. However, in the case of the septal rats, responding seems to be solely a function of the reinforcement density generated by the current schedule.

In this connection it is of interest to note that, while septal animals' performance on a given schedule is relatively insensitive to the effects of training on previous schedules, rats with hippocampal lesions, which also overrespond on DRL schedules, are particularly sensitive to prior training conditions (Schmaltz \& Isaacson, 1966; Ellen \& Aitken, in preparation ${ }^{1}$ ). This would suggest that the overresponding seen following these different lesions is based upon different mechanisms.

In addition, the failure of the septal rats to exhibit temporal discrimination (i.e., an increase in response probability, as measured by the IRTs/OP statistic, up to and beyond the required delay) on DRL 20, 30, 40, and 50 replicates and extends the generality of our previous observations (Ellen \& Aitken, 1971). In the present study, the response probability curve for septal animals generated by DRL $20,30,40$, and 50 remained constant for delays longer than $2 \mathrm{sec}$ since a previous response. Of particular interest was the fact that the response probabilities of the normal animals increased only in the range of 10-30 sec since the previous response on each of DRL $20,30,40$, and 50 . Since the range of delay in which the response probability increased did not covary with the schedule requirement, it would suggest that some process which decays with time since a previous response is implicated in the responding of normal animals but not in the septals. The nature of this process is at present unknown.

Two additional observations are worth emphasizing. On DRL 10 the IRTs/OP curves for septals and normals were identical in the range of unreinforced delays. However, in the range of reinforced delays, septals had a 
higher probability of responding than did normals. This would suggest an enhanced reactivity to reinforcing events-a phenomenon already quite well documented (e.g., Beatty \& Schwartzbaum, 1968; Carlson \& Cole, 1970; Zucker, 1965). Finally, it is of interest to note that, while the IRTs/OP curves for septals on DRL 20, 30,40 , and 50 are higher than those generated by DRL 10 for delays up to $10 \mathrm{sec}$ from a previous response, the curves for normals are lower than those generated by DRL 10 in this range. This would suggest that on DRL 20,30, 40, and 50, where there are relatively few reinforcements, septal animals fail to suppress responding during the first $10 \mathrm{sec}$ following a response, replicating a previous study (Ellen, Wilson, \& Powell, 1964). However, when there is an external event, such as a reinforcement, occurring fairly regularly, as is the case on DRL 10, then this failure to suppress in the first $10 \mathrm{sec}$ does not occur. In short, these findings lend further support to the view that septal animals fail to suppress responding in the absence of external stimuli (Ellen \& Butter, 1969) and, as a result, the failure of these animals to acquire a temporal discrimination on DRL is actually secondary to the loss of response suppression. for sucrose following septal lesions in the rat. Journal of Comparative \& Physiological Psychology, 1968, 65, 93-102.

Carlson, N. R., \& Cole, J. R. Enhanced alternation performance following septal lesions in mice. Journal of Comparative \& Physiological Psychology, 1970, 73, 157-161.

Ellen, P., \& Aitken, W. C., Jr. Absence of temporal discrimination following septal lesions. Psychonomic Science, 1971, 22, 129-131.

Ellen, P., \& Butter, J. External cue control of DRL performance in rats with septal lesions. Physiology \& Behavior, 1969, 4, $1-6$.

Ellen, P., DeLoache, C. H., \& Bonds, J. Time-shared control of a variety of psychological laboratories using the IBM-1800 data acquisition and control computer. Behavior Research Methods \& Instrumentation, 1972, 4, 81-85.

Ellen, P., Wilson, A. S., \& Powell, E. W. Septal inhibition and timing behavior in the rat. Experimental Neurology, 1964, 10, 120-132.

Pellegrino, L. J., \& Cushman, A. J. A stereotaxic atlas of the rat brain. New York: Appleton-Century-Crofts, 1967.

Schmaltz, L. W., \& Isaacson, R. L. The effect of preliminary training conditions upon DRL performance in the hippocampectomized rat. Physiology \& Behavior, 1966, 1, 175-182.

Staddon, J. E. R. Some properties of spaced responding in pigeons. Journal of the Experimental Analysis of Behavior, 1965, 8, 19-27.

Winer, B. J. Statistical principles in experimental design. New York: McGraw-Hill, 1962.

Zucker, I. Effect of lesions of the septal-limbic area on the behavior of cats. Journal of Comparative \& Physiological Psychology, 1965, 60, 344-352.

\section{NOTE}

\section{REFERENCES}

Anger, D. Dependence of interresponse times upon the relative reinforcement of different interresponse times. Journal of Experimental Psychology, 1956, 52, 145-161.

Beatty, W. W., \& Schwartzbaum, J. S. Consummatory behavior
1. Ellen, P., \& Aitken, W. C., Jr. Pretraining effects on the performance of rats with hippocampal lesions. Article in preparation.

(Received for publication August 21, 1972; revision received September 25,1972 .) 